Supporting information

\title{
Highly Active Zinc Sulfide Composite Microspheres: A Versatile Templates for Synthesis of a Family of Hollow Nanostructures of Sulfides
}

Chen-Yang Zhang $₫$, Wan-Ni Wang $\$$, Zhao-You Chu ${ }^{\ddagger}$, Hai-Sheng Qian ${ }^{\dagger, \S, *}$

$\uparrow$ School of Biomedical Engineering, Anhui Medical University, Hefei 230032, P. R. China

\$ School of Food and Biological Engineering, Hefei University of Technology, Hefei 230009, P. R. China

$\S$ Research and Engineering Center of Biomedical Materials, Anhui Medical University, Hefei 230032, P. R. China

Corresponding author. Email address: shqian@mail.ustc.edu.cn; shqian@hfut.edu.cn (H. Q.)

Number of pages: 14

Number of figures: 10

Number of tables: 2 


\section{Supporting Methods}

\subsection{Scalable synthesis of $A A-\left[Z n(O H)_{4}\right]^{2-}$ complex microspheres}

In a typical synthesis route, $7.2 \mathrm{mmol}$ of $\mathrm{CTAB}$ and $0.72 \mathrm{mmol}$ of $\mathrm{AA}$ were added into a $250 \mathrm{~mL}$ round-bottom flask and dissolved using $150 \mathrm{~mL}$ deionized water. Subsequently, $2.7 \mathrm{mmol}$ of $\mathrm{Zn}\left(\mathrm{NO}_{3}\right)_{2} \cdot 6 \mathrm{H}_{2} \mathrm{O}$ and $2.7 \mathrm{mmol}$ of HMTA were introduced into the solution with vigorously stirred for $30 \mathrm{~min}$ before being heated and maintained at $85^{\circ} \mathrm{C}$ for $10 \mathrm{~h}$. The resulting milky white suspension was washed three times with deionized water and absolute ethanol, and then dried at $50{ }^{\circ} \mathrm{C}$ for $24 \mathrm{~h}$. Finally, the AA- $\left[\mathrm{Zn}(\mathrm{OH})_{4}\right]^{2-}$ complex was obtained.

\subsection{Hyperthermic effect of the as-prepared samples under NIR irradiation}

To evaluate the photothermal conversion performance, $2.0 \mathrm{~mL}$ of the as-prepared $\mathrm{CuS}$ hollow nanosphere aqueous dispersions $\left(150 \mu \mathrm{g} \mathrm{mL}^{-1}\right)$ were irradiated by an 808 $\mathrm{nm}$ laser, and the temperature change of the aqueous dispersions was recorded every $10 \mathrm{~s}$ by a digital thermometer. 


\section{Supporting Figures}

Table S1. The reaction parameters of $\mathrm{SS}-\mathrm{M}_{\mathrm{x}} \mathrm{S}_{\mathrm{y}}(\mathrm{M}=\mathrm{Cu}, \mathrm{Ag}, \mathrm{Bi})$ hollow microspheres.

\begin{tabular}{cccc} 
Hollow nanosphere & Amount of thiourea & Solvent & Reaction temperature \\
\hline $\mathrm{CuS}$ & $0 \mathrm{mmol}$ & Deionized water & $25,45,65,85{ }^{\circ} \mathrm{C}$ \\
$\mathrm{Ag}_{2} \mathrm{~S}$ & $0 \mathrm{mmol}$ & Ethylene glycol & $130{ }^{\circ} \mathrm{C}$ \\
$\mathrm{Bi}_{2} \mathrm{~S}_{3}$ & $0.81 \mathrm{mmol}$ & Ethylene glycol & $130{ }^{\circ} \mathrm{C}$ \\
\hline
\end{tabular}


Table S2. The reaction parameters of $\mathrm{SS}_{-} \mathrm{Zn}_{\mathrm{x}} \mathrm{M}_{1-\mathrm{x}} \mathrm{S}(\mathrm{M}=\mathrm{Co}, \mathrm{Cd}, \mathrm{Ni})$ and $\mathrm{DS}-\mathrm{Zn}_{\mathrm{x}} \mathrm{M}_{1-\mathrm{x}} \mathrm{S}(\mathrm{M}=\mathrm{Mn}, \mathrm{Cd})$ hollow nanosphere.

\begin{tabular}{cccc}
\hline Hollow nanosphere & Amount of thiourea & Solvent & Reaction temperature \\
\hline $\mathrm{SS}-\mathrm{Zn}_{\mathrm{x}} \mathrm{Co}_{1-\mathrm{x}} \mathrm{S}$ & $16.2 \mathrm{mmol}$ & Deionized water & $150{ }^{\circ} \mathrm{C}$ \\
$\mathrm{SS}-\mathrm{Zn}_{\mathrm{x}} \mathrm{Cd}_{1-\mathrm{x}} \mathrm{S}$ & $16.2 \mathrm{mmol}$ & Ethylene glycol & $140{ }^{\circ} \mathrm{C}$ \\
$\mathrm{SS}-\mathrm{Zn}_{\mathrm{x}} \mathrm{Ni}_{1-\mathrm{x}} \mathrm{S}$ & $16.2 \mathrm{mmol}$ & Deionized water & $150{ }^{\circ} \mathrm{C}$ \\
$\mathrm{DS}-\mathrm{Zn}_{\mathrm{x}} \mathrm{Mn}_{1-\mathrm{x}} \mathrm{S}$ & $16.2 \mathrm{mmol}$ & Glycerinum + water & $160{ }^{\circ} \mathrm{C}$ \\
$\mathrm{DS}-\mathrm{Zn}_{\mathrm{x}} \mathrm{Cd}_{1-\mathrm{x}} \mathrm{S}$ & $40.5 \mathrm{mmol}$ & Deionized water & $140{ }^{\circ} \mathrm{C}$ \\
\hline
\end{tabular}




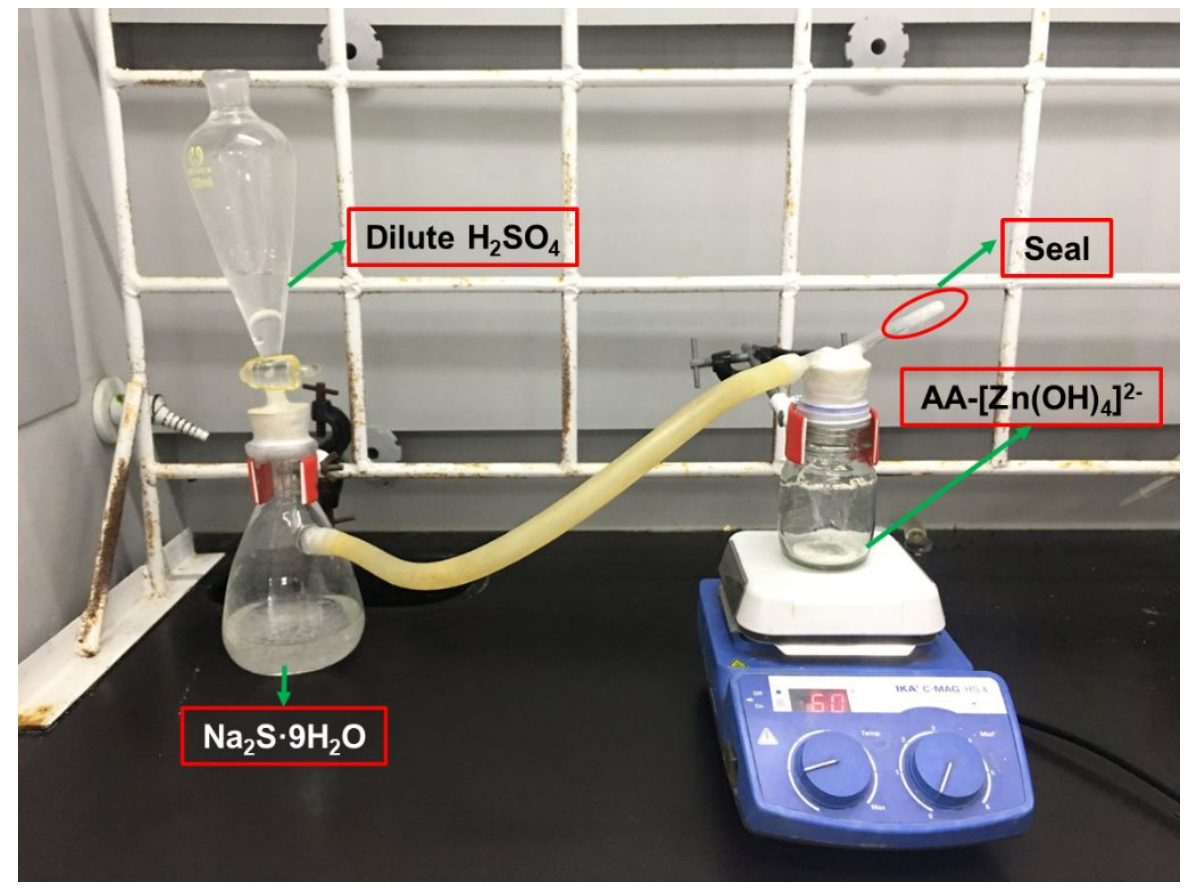

Figure S1. Photograph of the self-regulating gas sulfidation installation. 

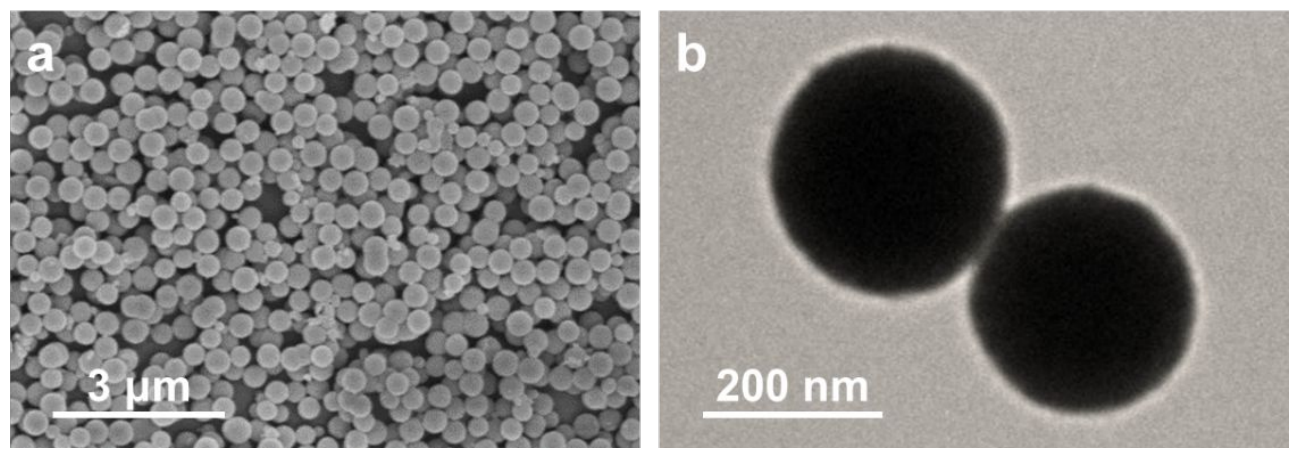

Figure S2. SEM (a) and TEM (b) images of as-prepared monodisperse AA- $\left[\mathrm{Zn}(\mathrm{OH})_{4}\right]^{2-}$ composite microspheres. 


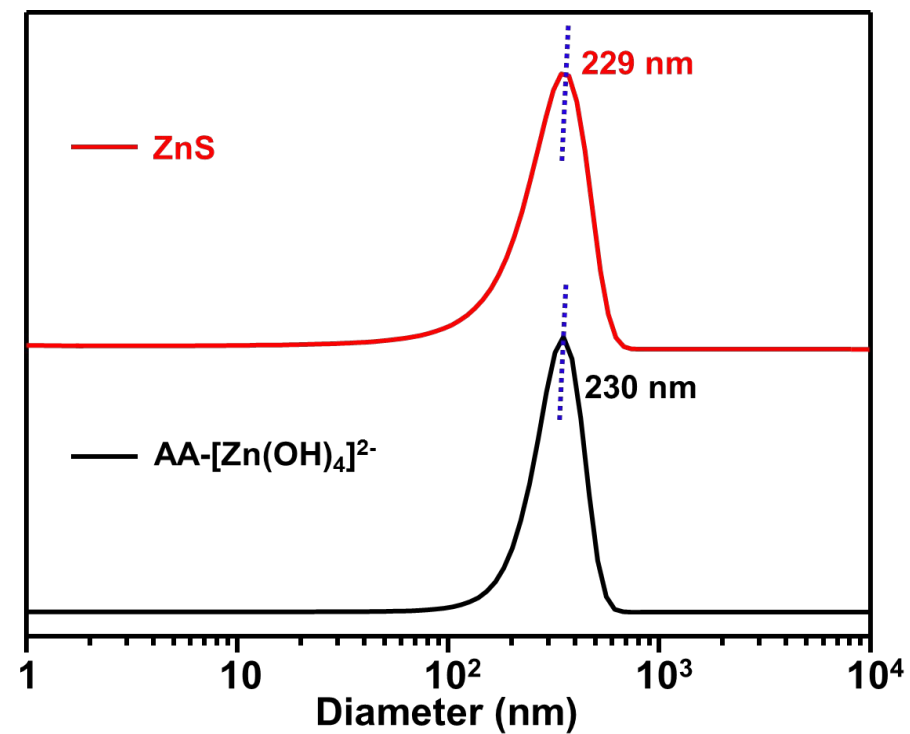

Figure S3. Particle size distribution of the as-prepared AA- $\left[\mathrm{Zn}(\mathrm{OH})_{4}\right]^{2-}$ complex microspheres and $\mathrm{ZnS}$ composite microspheres. 


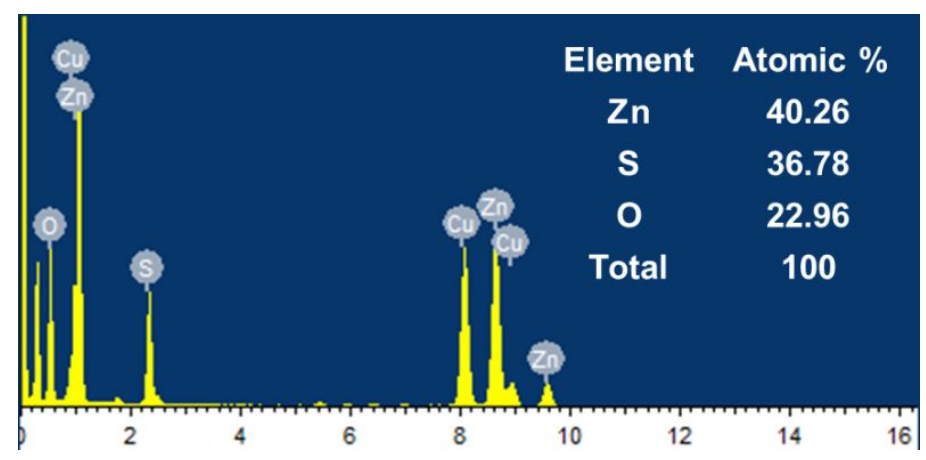

Figure S4. Energy dispersive X-ray spectra of the as-prepared $\mathrm{ZnS}$ composite microspheres. 


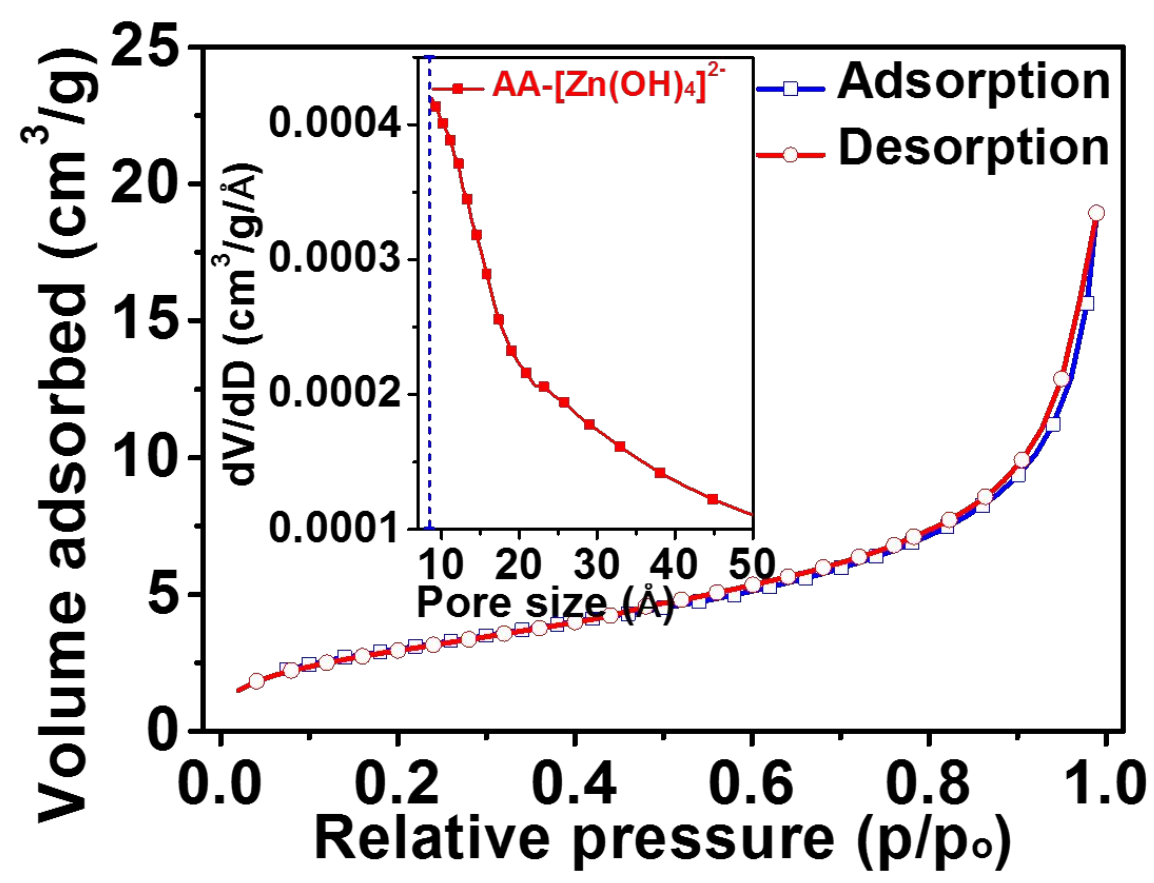

Figure S5. Nitrogen adsorption-desorption isotherms and pore-size distribution curves of the as-synthesized AA-[Zn(OH $\left.(\mathrm{OH})_{4}\right]^{2-}$ complex microspheres. 

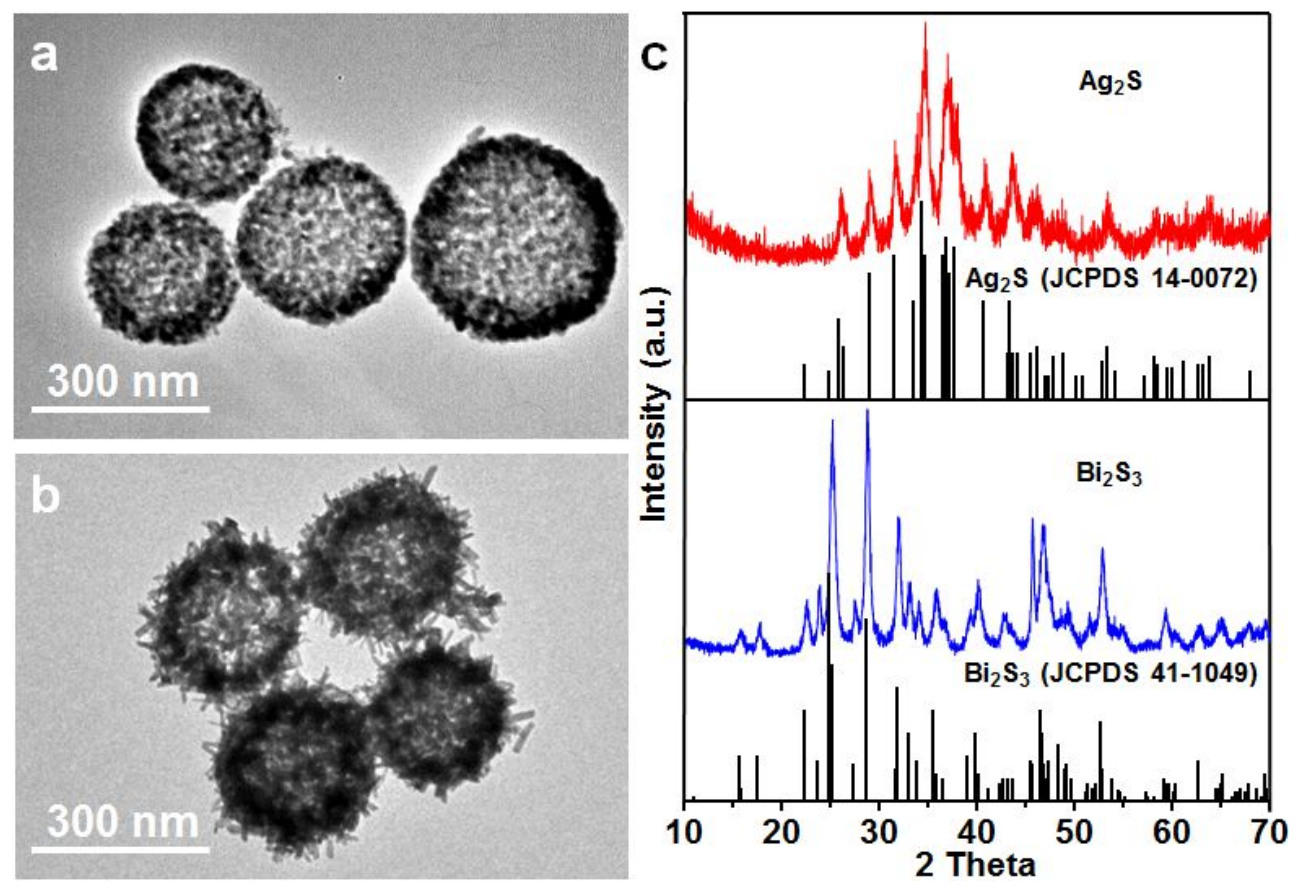

Figure S6. TEM images of as-obtained $\mathrm{Ag}_{2} \mathrm{~S}(\mathrm{a})$ and $\mathrm{Bi}_{2} \mathrm{~S}_{3}$ (b) hollow microspheres; (c) XRD patterns of as-obtained samples. 




Figure S7. TEM image of as-prepared $\mathrm{Cu}_{\mathrm{x}} \mathrm{Bi}_{1-\mathrm{x}} \mathrm{S}$ hollow microspheres. 



Figure S8. (a-d) STEM image and elemental mappings of S, Bi and $\mathrm{Cu}$ elements.

Scale bars are $300 \mathrm{~nm}$. 


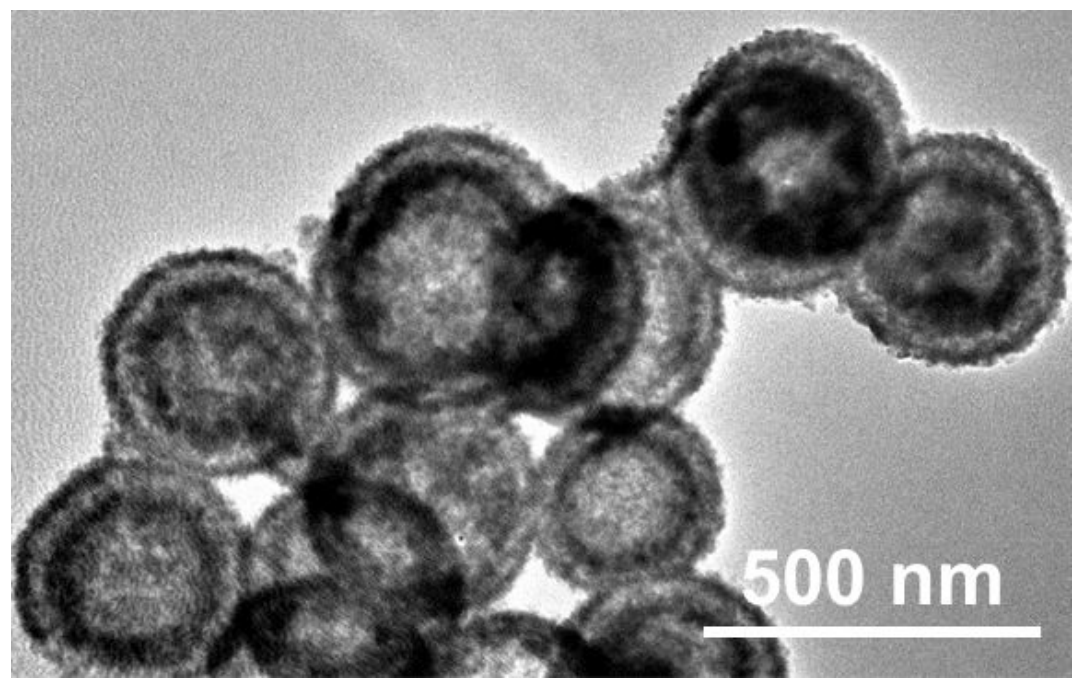

Figure S9. TEM image of DS- $\mathrm{Zn}_{\mathrm{x}} \mathrm{Cd}_{1-\mathrm{x}} \mathrm{S}$ hollow microspheres. 


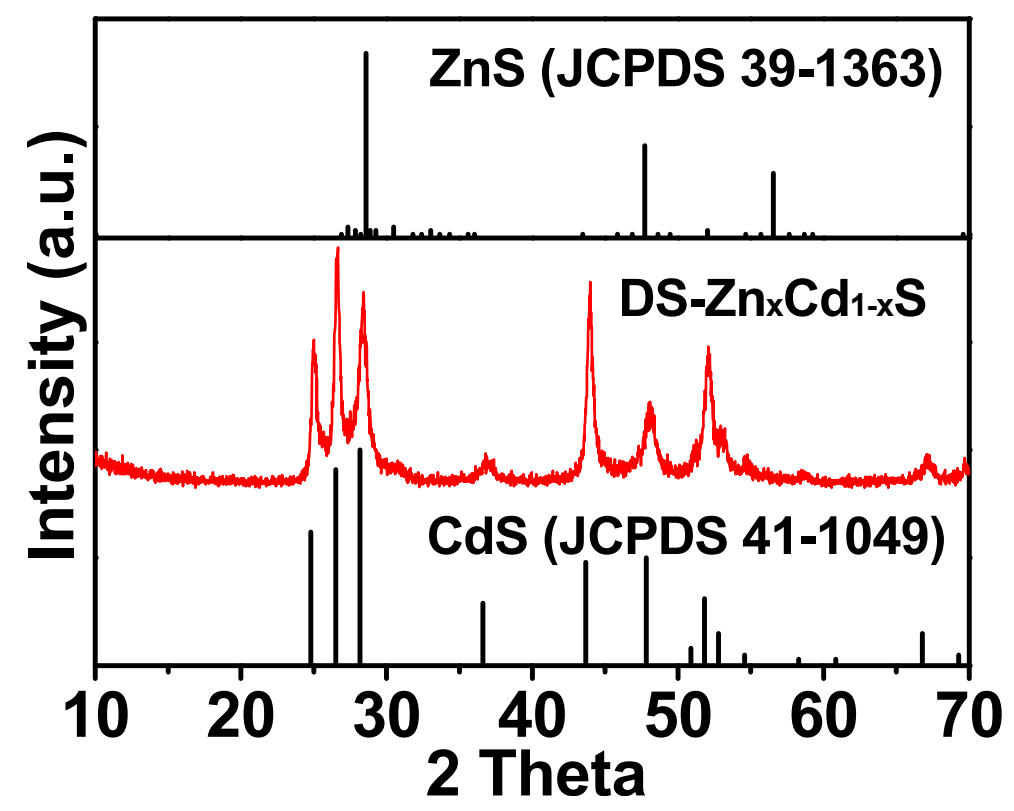

Figure S10. XRD patterns of the as-obtained DS- $\mathrm{Zn}_{\mathrm{x}} \mathrm{Cd}_{1-\mathrm{x}} \mathrm{S}$ hollow microspheres. 